\title{
Monotonicity in optimal mechanisms in general quasi-linear environments with a continuum of types
}

\section{Yu Chen ${ }^{1}$}

Received: 25 February 2018 / Accepted: 15 December 2018 / Published online: 31 December 2018 (c) The Author(s) 2018

\begin{abstract}
This paper provides conditions for the existence of optimal mechanism, in which the assignment rule takes the monotonic form and is determined independently of the transfer rule, in a principal-agent problem with a general quasi-linear environment. It is based on a complete characterization result of incentive-compatible mechanisms by monotonicity, envelope, and lower boundary individual rationality conditions. In particular, we present a general result for such an existence, which is also applicable to the scenarios without linear payoffs or regularity (lattice-related) conditions.
\end{abstract}

Keywords Monotonicity · Principal-agent problem · Optimal mechanism · Quasi-linear environment · Helly's selection principle

JEL Classification D82 - D86 · C62

\section{Introduction}

Monotonicity of assignment rules in optimal mechanisms has attracted much attention in the theory of mechanism design with quasi-linear environments. Normally, the assignment rule is determined independently of the transfer rule, which indicates a well-behaved property of the optimal mechanisms and carries significant analytical advantages. This property can further help construct the specific optimal mechanisms and analyze more specific issues under more concrete environments, such as nonlinear pricing, regulation, and auction. ${ }^{1}$

This paper provides general conditions for the existence of an optimal incentivecompatible mechanism, in which the assignment rule takes the monotonic form and

\footnotetext{
${ }^{1}$ See Appendix 3.1. in Laffont and Martimort (2009), among many others.
}

This work is supported by the National Natural Science Foundation of China (Grant no. 71673133).

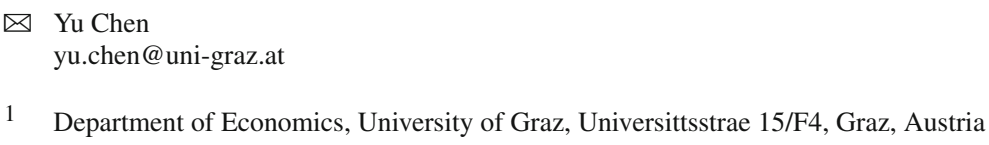


is determined independently of the transfer rule, in a general quasi-linear environment with a continuum of types. The general quasi-linear environment of our interests can cover a wide array of economic settings. Many applied mechanism design studies focus on linear payoffs. Nevertheless, we allow the agent's payoff to take the general quasi-linear form, since many other real-world scenarios, such as vertical contracting, nonlinear pricing, and labor contracts, do not exhibit linear payoff structures. We can further permit the assignment set to be more general by assuming a general quasilinear payoff. The assignment set can be multi-dimensional or even a set of functions; we only need a general order structure and a metric structure over it.

The existence of an optimal mechanism with monotonicity of assignment rule is significantly important. In many applications of mechanism design with simplified settings, it is possible to construct concrete optimal mechanisms or derive useful predication. None of these are relevant in a more general environment unless we can ensure that an optimal mechanism with monotonicity of assignment rule exists. It may also shed light on interesting extensions of the simple models.

In a general quasi-linear principal-agent environment, we completely characterize incentive-compatible and individual rational mechanisms by monotonicity, envelope, and lower boundary individual rationality conditions. Our characterization results are based on a set of mild, easy-to-check conditions on the primitives, so they can provide more concrete information about the (optimal) incentive-compatible mechanisms and make the original problems more tractable. We further construct an optimal mechanism in which the assignment rule takes the monotonic form and is determined independently of the transfer rule. Finally, we provide a set of lattice-related conditions and a set of topological conditions for the existence of such an optimal mechanism in terms of pointwise optimization. More importantly, we further show that a set of lattice-related conditions is even negligible for its general existence (not in terms of pointwise optimization). The extension of Helly's selection principle introduced by Chistyakov (2005) is useful for establishing the general existence result without these lattice-related conditions. This general existence result is of greater significance, especially in the scenarios without linear payoffs or those lattice-related conditions for pointwise optimization.

Ülkü (2013) provides a complete characterization of ex post incentive-compatible mechanisms to study the optimal combinatorial auction mechanism. His characterization result is based on a one-principal-multiple-agent situation but can naturally reduce to a single agent case. In his model, each assignment is simply a set of finite objects, whereas we consider a more general case. Beyond measurability, he imposes on the set of mechanisms an ad hoc restriction related to the integrability of the agents' expected payoffs under the given mechanisms. We can still adopt this restriction, but we also consider some easy-to-check, intuitive assumptions imposed on the primitives to substitute it. In his analysis, the principal only cares about her revenue maximization. In contrast, we allow the principal's payoff to be any Borel-measurable function. Finally, his analysis does not address the monotonicity of optimal mechanisms or general existence result of such optimal mechanisms.

This study is also related to a set of studies on general existence results for the optimal mechanism in a principal-agent problem. Carlier (2001) offers a general existence result for an adverse selection model with a continuum of types via $h$-convexity. His 
finding is also based on characterization result, but does not involve the monotonicity in optimal mechanisms. In contrast, this study provides easy-to-check assumptions and conditions for the monotonicity in optimal mechanisms. In a similar line of research, Page (1991, 1992) provide the general conditions for the existence of optimal deterministic mechanisms for principal-agent problems with both moral hazard and adverse selection. Kadan et al. (2017) examine the general conditions for the existence of optimal randomized mechanisms in principal-agent problems. However, due to their generality, none of these studies address the characterization and the monotonicity in optimal mechanisms.

\section{Model}

Consider a standard adverse selection model with one principal and one agent. The agent has a private type $\theta \in \Theta=[\underline{\theta}, \bar{\theta}] . \theta$ has a distribution function $F: \Theta \rightarrow[0,1]$ and an associated density function $f: \Theta \rightarrow \mathbb{R}_{+}$. The assignment for the agents is $x \in X$, where $X$ is a metric lattice endowed with a partial order, on which a strict partial order associated with the partial order is also defined. ${ }^{2}$ The monetary transfer for the agent is $t \in T \subseteq \mathbb{R}$. By the well-known revelation principle, it suffices to focus on direct mechanisms rather than all general mechanisms. A (direct) mechanism is a pair of Borel measurable functions $(\mathbf{x}, \mathbf{t})$, in which the assignment rule $\mathbf{x}: \Theta \rightarrow X$ specifies assignments to the agent for each type report profile, and the transfer rule $\mathbf{t}: \Theta \rightarrow T$ specifies a transfer to the agent for each type report profile.

The principal's payoff takes the quasi-linear form:

$$
u(x, \theta)-t,
$$

where $u: X \times \Theta \rightarrow \mathbb{R}$ is the payoff function from the principal's assignment. The agent's payoff takes the quasi-linear form:

$$
v(x, \theta)+t
$$

where $v: X \times \Theta \rightarrow \mathbb{R}$ is the agent's payoff function from the assignment. The agent has the reservation utility $r(\theta) \in \mathbb{R}$ for each $\theta \in \Theta$.

A mechanism $(\mathbf{x}, \mathbf{t})$ satisfies incentive compatibility (IC) if for all $\theta, \theta^{\prime} \in \Theta$, $v(\mathbf{x}(\theta), \theta)+\mathbf{t}(\theta) \geq v\left(\mathbf{x}\left(\theta^{\prime}\right), \theta\right)+\mathbf{t}\left(\theta^{\prime}\right)$. A mechanism $(\mathbf{x}, \mathbf{t})$ satisfies individual rationality (IR) if for all $\theta \in \Theta, v(\mathbf{x}(\theta), \theta)+\mathbf{t}(\theta) \geq r(\theta)$. Moreover, a mechanism (x, t) is feasible if it satisfies IC and IR. Thus, the principal's problem is

$$
\begin{cases}\max _{(\mathbf{x}, \mathbf{t})} & \int_{\Theta}(u(\mathbf{x}(\theta), \theta)-\mathbf{t}(\theta)) \mathrm{d} F(\theta) \\ \text { s.t. } & (\mathbf{x}, \mathbf{t}) \text { satisfies IC and IR. }\end{cases}
$$

A few assumptions are needed for further analysis.

\footnotetext{
2 For instance, if $X=\mathbb{R}$, the partial order is given by " $\geq$ " and the associated strict order is given by " $>$ ". If $X$ is a set of points, the partial order is given by " $\subseteq$ " and the associated strict order is given by " $\subset$ ".
} 
Assumption 1 (Absolute continuity) $v(x, \theta)$ is absolutely continuous ${ }^{3}$ in $\theta$ for all $x$.

Assumption 2 (Differentiability) $v(x, \theta)$ is differentiable in $\theta$ for all $x \cdot v_{\theta}(x, \theta) \equiv$ $\frac{\partial v(x, \theta)}{\partial \theta}$ exists for all $x \in X$ (right differentiable at $\underline{\theta}$ and left differentiable at $\bar{\theta}$ ).

Assumption 3 (Integrability) $\mathbf{x}$ is such that $v_{\theta}(\mathbf{x}(\cdot), \cdot)$ is Riemann integrable on $\Theta$.

Assumption 4 (Single-crossing property) $v(x, \theta)$ has strictly increasing differences in $x$ and $\theta$; that is, for each $x^{\prime \prime}>x^{\prime}$ in $X$ and each $b>a$ in $\Theta^{4}$

$$
v\left(x^{\prime \prime}, b\right)-v\left(x^{\prime}, b\right)>v\left(x^{\prime \prime}, a\right)-v\left(x^{\prime}, a\right) .
$$

Assumption 5 (Measurability) $u(x, \theta)$ is Borel measurable in $x$ and $\theta$.

Although the scenarios of mechanism design with linear payoffs clearly satisfy all the aforementioned assumptions, there still are remarkable scenarios of mechanism design naturally without linear payoffs. A few examples without linear payoffs are presented as follows. In each of the examples, Assumptions 1-5 are satisfied. ${ }^{5}$

Example 1 (Vertical contracting) In a similar setting of vertical contracting as in Dequiedt and Martimort (2015), an upstream manufacturer (principal) contracts with a downstream retailer (agent). The manufacturer sells to the retailer a quantity $x \in X \subseteq[0, \infty)$ of an essential input, at price $p \in[0, \infty)$. The retailer will transform this input, with a one-to-one Leontief technology, into $x$ units of his final good on the downstream market at a cost $c x^{2}$. The retailer privately knows $c \in[\underline{c}, \bar{c}]$ as the transformation technology efficiency. The retailer's revenue is $x P(x)-c x^{2}$, where $P$ denotes the inverse demand function on the downstream market and is continuous in $x$. The retailer has the payoff $x P(x)-c x^{2}-p$. We further define $\theta:=-c, t:=-p$, and then the retailer's payoff becomes $v(x, \theta)+t$, where $v(x, \theta)=x P(x)+\theta x^{2}$. The manufacturer has a payoff $p-c(x)$, where $c(x)$ represents the manufacturing cost and is continuous in $x$.

Example 2 (Nonlinear pricing) In a similar setting of nonlinear pricing as in Maskin and Riley (1984), a monopolist (principal) sells a single product with quantity $x \in$ $X \subseteq[0, \infty)$ to a buyer (agent) with a private taste $\theta \in[\underline{\theta}, \bar{\theta}]$. The inverse demand function of the buyer takes a linear form: $p(x, \theta)=\theta-\beta(\theta) q$, where $\beta: \Theta \rightarrow \mathbb{R}$ is nonnegative, differentiable, and decreasing. $\beta^{\prime}$ is clearly bounded on $[\theta, \bar{\theta}]$. The buyer also needs to pay $w \in[0, \infty)$ to purchase the product, and then he has the payoff $v(x, \theta)-w$, where $v(x, \theta)=\int_{0}^{x} p(q, \theta) \mathrm{d} q$. We can define $t:=-w$. We can find $v_{\theta}(x, \theta)=\int_{0}^{x} p_{\theta}(q, \theta) \mathrm{d} q=\int_{0}^{x} 1-\beta^{\prime}(\theta) q \mathrm{~d} q$, which is nonnegative. The principal's payoff function is given by $w-c(x)$, where $c(x)$ represents the production cost and is continuous in $x$.

\footnotetext{
3 Note that continuous differentiability is a form of absolute continuity, which is frequently adopted in many applications.

4 Under Assumption 2, Assumption 4 is equivalent to saying that $v_{\theta}(x, \theta)$ is strictly increasing in $x$.

5 In subsequent analysis, we will see that Assumption 3 can be implied by two easy-to-check Assumptions 6 and 7 , which hold in these examples.
} 
Example 3 (Optimal labor contracts) In light of Hart (1983), we consider that an employer (principal) specifies an output (task) $x \in X \subseteq[0, \infty)$ and wage $t \in[0, \infty)$ for an employee (agent). The employee has a production function $x(l, \theta)=\theta l$, where $l \in L \subseteq[0, \infty)$ is his labor input and his marginal product $\theta \in[\underline{\theta}, \bar{\theta}] \subseteq(0, \infty)$. But the employer can only observe the final output. Both $l$ and $\theta$ are private information of the employee. The employee's payoff is $v(x, \theta)+t$, where $v(x, \theta)=-\left(\frac{x}{\theta}\right)^{2}$ and $\left(\frac{x}{\theta}\right)^{2}$ denotes the employee's input cost. We can find $v_{\theta}(x, \theta)=\frac{2 x^{2}}{\theta^{3}}$, which is positive. The employer's payoff function is given by $B(x)-t$, where $B(x)$ represents the revenue of the employer from the output and is continuous in $x$.

We also define the agent's truth-telling payoff with respect to $(\mathbf{x}, \mathbf{t})$ as:

$$
\bar{V}(\theta ; \mathbf{x}, \mathbf{t})=v(\mathbf{x}(\theta), \theta)+\mathbf{t}(\theta)
$$

when the agent truthfully reports his own type $\theta$.

\section{Characterization results}

We first provide a complete characterization of feasible mechanisms with monotonic assignment rules under our assumptions in general quasi-linear environments.

Proposition 1 Under Assumptions 1-4, a mechanism (x, t) is IC if and only iffor each $a, b \in \Theta$,

$$
\bar{V}(b ; \mathbf{x}, \mathbf{t})-\bar{V}(a ; \mathbf{x}, \mathbf{t})=\int_{a}^{b} v_{\theta}(\mathbf{x}(s), s) \mathrm{d} s
$$

An IC mechanism $(\mathbf{x}, \mathbf{t})$ is IR if and only if

$$
\bar{V}(\underline{\theta} ; \mathbf{x}, \mathbf{t}) \geq r(\underline{\theta})
$$

(lower boundary IR)

The monotonicity condition is solely relevant for the assignment rule under the single-crossing assumption and it renders a well-behaved property of the assignment rule that it must be increasing with respect to the agent's type. Meanwhile, the envelope condition states that any difference between the agent's truth-telling payoffs caused by his types equals the integral of the marginal payoff from assignments with respect to his type within the two types. ${ }^{6}$ Joint with the other two conditions, there is no loss of generality from restricting attention to the lower boundary IR condition for the agent simply with the lowest type from the original IR condition.

\footnotetext{
6 Moreover, Eq. (6) in the proof of Proposition 1, as the corresponding differential condition, uncovers how the agent's truth-telling payoff changes with respect to his own type as a parameter. This equation relates to the envelope theorem as mentioned in Milgrom and Segal (2002).
} 
First, note that absolute continuity in Assumption 1 may be implied by other easy-tocheck conditions, given the differentiability of $v(x, \cdot)$. The frequently used condition would be: Assumption $1^{\prime} v(x, \theta)$ is increasing in $\theta$ for all $x$. We know that $v(x, \cdot)$ is differentiable and increasing. Thus, $v_{\theta}(x, \cdot)$ is Lebesgue integrable and, therefore, Theorem 1 in Koliha (2006) implies the absolute continuity of $v(x, \cdot)$.

Assumption 3 is also adopted by Ülkü (2013); however, Assumption 3 imposes somewhat ad hoc conditions directly on the assignment rule, which is the choice variable of (P1). We can introduce two easy-to-check, intuitive conditions-Assumptions 6 and 7 below-to substitute Assumption 3. They simply impose mild conditions on the primitives. In our subsequent analysis, Assumptions 6 and 7 together with other assumptions imply Assumption 3. In Corollary 1 below, we show that all the subsequent results hold if we replace Assumption 3 with Assumptions 6 and 7.

Assumption 6 (Boundedness) $v_{\theta}(x, \cdot)$ is bounded for each $x$.

Assumption 7 (Continuity or increasingness) $v_{\theta}(x, \theta)$ is continuous or monotonic in $\theta$ for all $x$.

Corollary 1 If we replace Assumption 3 with Assumptions 6 and 7, the result in Proposition 1 still holds.

The characterization result of feasible mechanisms presented in Proposition 1 or Corollary 1 constitutes a starting point for our further analysis in this paper. Although such a result is not conceptually new, its main merit is embodied in its greatest generality within the scope of monotonic assignment rules given a continuum of types. In most standard results of the existing literature concerning the characterization of feasible mechanisms with monotonic assignment rules given a continuum of types, e.g., in Stole (2001), the set of assignments is normally assumed to be $\mathbb{R}_{+}$; the direct mechanisms are assumed to be compact-valued; the payoff functions of both parties are at least twice continuously differentiable in both $x$ and $\theta$; and the single-crossing property is presented with differentiability of the agent's payoff function. ${ }^{7}$ By contrast, our analysis allows the set of assignments to be a metric lattice. We can avoid imposing ad hoc assumptions on the direct mechanisms beyond measurability. We only require differentiability of the agent's payoff function over $\theta$ but not over $x$. The principal's payoff function can be any Borel-measurable function. Moreover, typedependent outside option is permitted in our analysis.

Furthermore, based on Proposition 1, we can construct an optimal mechanism in which optimal assignment rule $\mathbf{x}^{*}$ can be independently determined, and optimal transfer rule $\mathbf{t}^{*}$ is subsequently determined by $\mathbf{x}^{*}$. Moreover, the principal's optimal objective value is uniquely determined by $\mathbf{x}^{*}$ independent of $\mathbf{t}^{*}$.

\footnotetext{
7 Some standard textbooks only focus on the environment with the agent's payoff linear in either his type or his assignment. For instance, see Appendix 3.1 in Laffont and Martimort (2009) or Section 2.3.3 in Bolton and Dewatripont (2005).
} 
Proposition 2 Under Assumptions 1-5, a mechanism $\left(\mathbf{x}^{*}, \mathbf{t}^{*}\right)$ is the optimal mechanism solving $(P 1)$ if and only if $\left(\mathbf{x}^{*}, \mathbf{t}^{*}\right)$ solves $(P 1 ')$ :

$$
\max _{(\mathbf{x}, \mathbf{t})} \int_{\Theta}\{u(\mathbf{x}(\theta), \theta)+(\Phi(\mathbf{x}(\theta), \theta)-\bar{V}(\underline{\theta} ; \mathbf{x}, \mathbf{t}))\} \mathrm{d} F(\theta)
$$

s.t. $(\mathbf{x}, \mathbf{t})$ satisfies the monotonicity, envelope, and lower boundary IR conditions,

where $\Phi(\mathbf{x}(\theta), \theta)=v(\mathbf{x}(\theta), \theta)-\frac{1-F(\theta)}{f(\theta)} v_{\theta}(\mathbf{x}(\theta), \theta)$.

In particular, the problem $(\mathrm{P} 1)$ admits a solution $\left(\mathbf{x}^{*}, \mathbf{t}^{*}\right)$ satisfying: $\mathbf{x}^{*}$ solves

$$
\max _{\mathbf{x} \text { satisfies the monotonicity condition }} \int_{\Theta}\{u(\mathbf{x}(\theta), \theta)+\Phi(\mathbf{x}(\theta), \theta)-r(\underline{\theta})\} \mathrm{d} F(\theta),
$$

and

$$
\mathbf{t}^{*}(\theta)=\int_{\underline{\theta}}^{\theta} v_{\theta}\left(\mathbf{x}^{*}(s), s\right) \mathrm{d} s-v\left(\mathbf{x}^{*}(\theta), \theta\right)+r(\theta),
$$

whenever for all $\theta, \mathbf{t}(\theta)=\int_{\theta}^{\theta} v_{\theta}(\mathbf{x}(s), s) \mathrm{d} s-v(\mathbf{x}(\theta), \theta)+r(\theta) \in T .{ }^{8}$ Moreover, $(\mathrm{P} 1)$ and $(\mathrm{P} 2)$ bring the same optimal value to the principal.

\section{Monotonicity of optimal mechanisms}

Based on the preceding characterization results, we can establish further existence results of the optimal mechanisms. Notice that these results will also ensure the existence of optimal mechanisms with the monotonicity in assignment rules. Therefore, the existence results can help further construct concrete optimal mechanisms in different scenarios. To establish the existence results, we need to introduce a few additional assumptions below. They are mild and easily satisfied in many applications, including Examples 1-3.

Assumption $8 v_{\theta}(x, \theta)$ is Borel-measurable in $\theta$ for each $x$.

Assumption $9 v(x, \theta)$ and $u(x, \theta)$ are upper semicontinuous in $x$ for each $\theta$, and $v_{\theta}(x, \theta)$ is lower semicontinuous in $x$ for each $\theta$.

Assumption $10 X$ is a compact subset of a normed vector space. ${ }^{9}$

In many applications of mechanism design, it is useful to find a version of the existence of optimal mechanisms through pointwise maximization of the principal's objective function. Let

$$
g(x, \theta):=u(x, \theta)+\Phi(x, \theta)-r(\underline{\theta})
$$

\footnotetext{
8 If $T=\mathbb{R}$, this will always hold.

9 A typical example is any compact set in a Euclidean space.
} 
and

$$
G(\mathbf{x}):=\int_{\Theta} g(\mathbf{x}(\theta), \theta) \mathrm{d} F(\theta) .
$$

Proposition 3 Under Assumptions 1-5 and 8-10, any mechanism $\mathbf{x}$ satisfying

$$
\mathbf{x}(\theta) \in \arg \max _{x \in X} g(x, \theta)
$$

and $\mathbf{x}(\theta)$ is increasing solves (P2). Such a mechanism exists if

(1) $X$ is a compact metric lattice,

(2) $u(x, \theta)+\Phi(x, \theta)$ is supermodular in $x$ for all $\theta,{ }^{10}$ and,

(3) $u(x, \theta)+\Phi(x, \theta)$ has increasing difference in $x$ and $\theta$.

Proposition 3 extends the regularity condition in the case with the linear payoff of the agent, ${ }^{11}$ which is adopted as the conventional technique in many previous studies. Proposition 3 imposes a few lattice-related conditions to guarantee the monotonicity of the optimal mechanism.

The hypotheses (2) and (3) in Proposition 3 may not always be easy to satisfy, especially in the scenario of mechanism design without linear payoffs. These two hypotheses also rely on both the distribution of the type and the payoff functions. For instance, it is difficult to check them in Examples 1-3. In addition, the relevant conditions on $u(x, \theta)+\Phi(x, \theta)$ normally have no clear economic meaning.

In fact, we find that the monotonicity of the optimal mechanism does not require these lattice-related conditions. This is summarized in Theorem 1 below. We can use the extension of Helly's selection principle in Chistyakov (2005) to show that the set of increasing assignment rules is sequentially compact with the topology of pointwise convergence. It is an important foundation for the general existence of optimal mechanisms.

Theorem 1 Under Assumptions 1- 5, and 8-10, there exists a solution to (P2) and then (P1).

By avoiding checking or imposing those lattice-related conditions required in Proposition 3, Theorem 1 has analytical advantage beyond the conventional technique dealing with the monotonic assignment rules given a continuum of types. Theorem 1 will be applicable to more scenarios of mechanism design, especially the scenarios of mechanism design without linear payoffs or the hypotheses (2) and (3) in Proposition 3. In those contexts, the conventional technique concerning pointwise maximization fails, but our results can still help establish the monotonicity of optimal mechanisms as a significant qualitative property. Furthermore, the optimal control technique ${ }^{12}$ is applicable to solving the general maximization problem in which the monotonicity may facilitate finding a tractable solution.

\footnotetext{
10 Note that it is always supermodular if $X$ is one dimensional or totally ordered.

11 Normally, the regularity condition says that $\frac{F(\theta)-1}{f(\theta)}$ is increasing in $\theta$. It may also apply when $v(x, \theta)$ takes the form $v(x, \theta)=\theta h(x)$, for some function $h: X \rightarrow \mathbb{R}$.

12 For instance, see Guesnerie and Laffont (1984) among others.
} 
Examples 1-3 will survive our general conditions, as long as we assume $X$ to be a compact set in $\mathbb{R}$. We do not have to additionally impose the lattice-related conditions required in Proposition 3. Let us take Example 3 as an instance. It is natural to assume the set of labor input $L=[0, \bar{l}]$ and, therefore, $X$ is also a closed interval in $[0, \infty) \cdot v(x, \theta)=-\left(\frac{x}{\theta}\right)^{2}$ is continuous in $x$ and differentiable and increasing in $\theta$. $v_{\theta}(x, \theta)=\frac{2 x^{2}}{\theta^{3}}$ is clearly bound for each $x$ and continuous. Single-crossing property also holds, since $v_{\theta x}(x, \theta)=\frac{4 x}{\theta^{3}}$. The employer's payoff is continuous in $x$ and $\theta$ does not enter the payoff function. Now all necessary conditions for Theorem 1 are satisfied, and we can conclude that the optimal mechanism in this case must exist and it can achieve a monotonic assignment rule. By contrast, if we intend to use Proposition 3, we need to additionally impose the "regularity condition" requiring that $\frac{2(F(\theta)-1)}{f(\theta) \theta^{3}}-\frac{1}{\theta^{2}}$ is increasing in $\theta$. This assumption may be strong or difficult-to-check, and it has no clear economic meaning.

Acknowledgements Open access funding provided by University of Graz. I would like to thank Christoph Kuzmics, Michael Greinecker, Nian Yang, Olga Gorelkina, Ran Shao, Desu Liu, Robert Becker, and Yong Sui for helpful suggestions and comments. I am solely responsible for any errors.

Open Access This article is distributed under the terms of the Creative Commons Attribution 4.0 International License (http://creativecommons.org/licenses/by/4.0/), which permits unrestricted use, distribution, and reproduction in any medium, provided you give appropriate credit to the original author(s) and the source, provide a link to the Creative Commons license, and indicate if changes were made.

\section{Appendix: All Proofs}

Proof of Proposition 1 First, assume that a given mechanism $(\mathbf{x}, \mathbf{t})$ is IC. Thus, for any $\theta \in(\underline{\theta}, \bar{\theta})$, and $\delta>0$ satisfying $\theta+\delta \in[\underline{\theta}, \bar{\theta}]$, the IC condition is clearly equivalent to

$$
\bar{V}(\theta+\delta ; \mathbf{x}, \mathbf{t})-\bar{V}(\theta ; \mathbf{x}, \mathbf{t}) \geq v(\mathbf{x}(\theta), \theta+\delta)-v(\mathbf{x}(\theta), \theta) .
$$

Reversing $\theta+\delta$ and $\theta$ in (1) and combining (1), we have

$$
\bar{V}(\theta+\delta ; \mathbf{x}, \mathbf{t})-\bar{V}(\theta ; \mathbf{x}, \mathbf{t}) \leq v(\mathbf{x}(\theta+\delta), \theta+\delta)-v(\mathbf{x}(\theta+\delta), \theta)
$$

(1) and (2) imply that

$$
v(\mathbf{x}(\theta), \theta+\delta)-v(\mathbf{x}(\theta), \theta) \leq v(\mathbf{x}(\theta+\delta), \theta+\delta)-v(\mathbf{x}(\theta+\delta), \theta)
$$

Now suppose that the monotonicity condition does not hold; that is, $\mathbf{x}(\theta)$ is not increasing in $\theta$. Thus, Assumption 5 implies that for some $\theta \in(\underline{\theta}, \bar{\theta})$, and some $\delta>0$ satisfying $\theta+\delta \in[\underline{\theta}, \bar{\theta}]$,

$$
v(\mathbf{x}(\theta), \theta+\delta)-v(\mathbf{x}(\theta), \theta)>v(\mathbf{x}(\theta+\delta), \theta+\delta)-v(\mathbf{x}(\theta+\delta), \theta) .
$$

This contradicts (3). Hence, the monotonicity condition follows. 
Next we prove the envelope condition. First we show that $\bar{V}(\cdot ; \mathbf{x}, \mathbf{t})$ is differentiable in $\theta$ almost everywhere. By Assumptions 1 and $2, v(x, \cdot)$ is differentiable ${ }^{13}$ and absolutely continuous. By (1), $\bar{V}(\theta+\delta ; \mathbf{x}, \mathbf{t})-\bar{V}(\theta ; \mathbf{x}, \mathbf{t}) \geq 0$, since $v(\mathbf{x}(\theta), \theta+\delta)-$ $v(\mathbf{x}(\theta), \theta) \geq 0$ by Assumption 2. Thus, by (2),

$$
|\bar{V}(\theta+\delta ; \mathbf{x}, \mathbf{t})-\bar{V}(\theta ; \mathbf{x}, \mathbf{t})| \leq|v(\mathbf{x}(\theta+\delta), \theta+\delta)-v(\mathbf{x}(\theta+\delta), \theta)| .
$$

Since $v(x, \cdot)$ is absolutely continuous, the inequality above implies that $\bar{V}(\cdot ; \mathbf{x}, \mathbf{t})$ is also absolutely continuous; then $\bar{V}(\cdot ; \mathbf{x}, \mathbf{t})$ is differentiable in $\theta$ almost everywhere.

Thus, consider the subset $E$ of $\Theta$ such that $\bar{V}(\cdot ; \mathbf{x}, \mathbf{t})$ is differentiable in $\theta$. Let $\delta>0$ satisfying $\theta+\delta \in[\underline{\theta}, \bar{\theta}]$. Then, by (1), we have

$$
\begin{aligned}
\lim _{\delta \rightarrow 0} \frac{\bar{V}(\theta+\delta ; \mathbf{x}, \mathbf{t})-\bar{V}(\theta ; \mathbf{x}, \mathbf{t})}{\delta} & \geq \lim _{\delta \rightarrow 0} \frac{v(\mathbf{x}(\theta), \theta+\delta)-v(\mathbf{x}(\theta), \theta)}{\delta} \\
& =v_{\theta}(\mathbf{x}(\theta), \theta)
\end{aligned}
$$

(2) implies that

$$
\begin{aligned}
\lim _{\delta \rightarrow 0} \frac{\bar{V}(\theta ; \mathbf{x}, \mathbf{t})-\bar{V}(\theta-\delta ; \mathbf{x}, \mathbf{t})}{\delta} & \leq \lim _{\delta \rightarrow 0} \frac{v(\mathbf{x}(\theta), \theta)-v(\mathbf{x}(\theta), \theta-\delta)}{\delta} \\
& =v_{\theta}(\mathbf{x}(\theta), \theta)
\end{aligned}
$$

Thus, by (4) and (5), we conclude that $\frac{\partial \bar{V}(\theta ; \mathbf{x}, \mathbf{t})}{\partial \theta}=v_{\theta}(\mathbf{x}(\theta), \theta)$ on $E$.

Next, by Assumption 3, $v_{\theta}(\mathbf{x}(\cdot), \cdot)$ is also Lebesgue integrable on $\Theta_{i}$, since $\Theta_{i}$ is a compact interval on $\mathbb{R}$. Moreover,

$$
\frac{\partial \bar{V}(\theta ; \mathbf{x}, \mathbf{t})}{\partial \theta}=v_{\theta}(\mathbf{x}(\theta), \theta), \quad \text { a.e. } \theta \in \Theta
$$

Hence, the envelope condition holds due to (6).

Second, assume that a given mechanism $(\mathbf{x}, \mathbf{t})$ satisfies the monotonicity and envelope conditions. Thus,

$$
\begin{aligned}
& \bar{V}(b ; \mathbf{x}, \mathbf{t})-\bar{V}(a ; \mathbf{x}, \mathbf{t}) \\
& \quad=\int_{a}^{b} v_{\theta}(\mathbf{x}(\theta), \theta) \mathrm{d} \theta \geq \int_{a}^{b} v_{\theta}(\mathbf{x}(a), \theta) \mathrm{d} \theta \\
& \quad=v(\mathbf{x}(a), b)-v(\mathbf{x}(a), a)
\end{aligned}
$$

Thus, the IC condition is satisfied, since it is equivalent to Eq. (7).

Lastly, based on the envelope condition, we only need to address the IR condition with the lower boundary IR condition. First, the lower boundary IR condition directly follows from IR condition. On the other hand, we take $b=\theta$ and $a=\underline{\theta}$ in the envelope

\footnotetext{
13 It is trivial to address one-sided differentiability at the two boundary points throughout the proof. Thus, we do not specifically address it in this proof.
} 
condition, and combine it with the lower boundary IR condition and Assumption 2, then the IR condition follows.

Proof of Corollary 1 Compared with the proof of Proposition 1, it suffices to show that Assumptions 6 and 7, joint with other assumptions, imply Assumption 3. First note that the monotonicity condition is proven without Assumption 3 under any given IC mechanism. Moreover, $v_{\theta}(x, \cdot)$ is continuous or increasing ${ }^{14}$ for all $x$ by Assumption 7. Thus, given increasing $\mathbf{x}(\cdot)$ and the single-crossing property, $v_{\theta}(x(\cdot), \cdot)$ must be increasing and therefore only has at most countable discontinuities. By Assumption 6 and Theorem 14.5 in Apostol (1974), $v_{\theta}(\mathbf{x}(\cdot), \cdot)$ is Riemann integrable on $\Theta$ for any $\mathbf{x}$.

Proof of Proposition 2 First rewrite the principal's objective function as follows

$$
\begin{aligned}
U(\mathbf{x}, \mathbf{t}) \equiv & \int_{\Theta} u(\mathbf{x}(\theta), \theta) \mathrm{d} F(\theta)+\int_{\Theta} v(\mathbf{x}(\theta), \theta) \mathrm{d} F(\theta) \\
& -\int_{\Theta}(v(\mathbf{x}(\theta), \theta)+\mathbf{t}(\theta)) \mathrm{d} F(\theta) .
\end{aligned}
$$

Let $I:=\int_{\Theta}(v(\mathbf{x}(\theta), \theta)+\mathbf{t}(\theta)) \mathrm{d} F(\theta)$. According to the definition of the agent's truth-telling payoff, we have

$$
\begin{aligned}
I= & \int_{\Theta} \bar{V}(\underline{\theta} ; \mathbf{x}, \mathbf{t}) \mathrm{d} F(\theta) \\
& +\int_{\Theta}\left[\int_{\underline{\theta}}^{\theta} \frac{\partial \bar{V}(s ; \mathbf{x}, \mathbf{t})}{\partial \theta} \mathrm{d} s\right] \mathrm{d} F(\theta)
\end{aligned}
$$

Changing the order of integration and integrating by parts, we have

$$
\begin{aligned}
I & =\int_{\Theta} \bar{V}(\underline{\theta} ; \mathbf{x}, \mathbf{t}) \mathrm{d} F(\theta)+\int_{\Theta}(1-F(\theta)) v_{\theta}(\mathbf{x}(\theta), \theta) \mathrm{d} \theta \\
& =\int_{\Theta} \bar{V}(\underline{\theta} ; \mathbf{x}, \mathbf{t}) \mathrm{d} F(\theta)+\int_{\Theta} \frac{1-F(\theta)}{f(\theta)} v_{\theta}(\mathbf{x}(\theta), \theta) \mathrm{d} F(\theta)
\end{aligned}
$$

So the principal's objective function is given by

$$
U(\mathbf{x}, \mathbf{t})=\int_{\Theta}\{u(\mathbf{x}(\theta), \theta)+(\Phi(\mathbf{x}(\theta), \theta)-\bar{V}(\underline{\theta} ; \mathbf{x}, \mathbf{t}))\} \mathrm{d} F(\theta)
$$

Thus, (P1) is clearly equivalent to (P1') due to Proposition 1.

14 Similar argument applies if $v_{\theta}(x, \cdot)$ is decreasing. 
Next, the envelope and lower boundary IR conditions give

$$
\begin{aligned}
r(\theta) & \leq \bar{V}(\theta ; \mathbf{x}, \mathbf{t})-\int_{\underline{\theta}}^{\theta} v_{\theta}(\mathbf{x}(s), s) \mathrm{d} s \\
& =v(\mathbf{x}(\theta), \theta)+\mathbf{t}(\theta)-\int_{\underline{\theta}}^{\theta} v_{\theta}(\mathbf{x}(s), s) \mathrm{d} s
\end{aligned}
$$

Thus, if the principal can select the transfer rule

$$
\mathbf{t}(\theta)=\int_{\underline{\theta}}^{\theta} v_{\theta}(\mathbf{x}(s), s) \mathrm{d} s-v(\mathbf{x}(\theta), \theta)+r(\theta),
$$

she satisfies both the envelope and lower boundary IR conditions, obtaining

$$
\int_{\Theta} \bar{V}(\underline{\theta} ; \mathbf{x}, \mathbf{t}) \mathrm{d} F(\theta)=r(\underline{\theta}),
$$

which is the best possible value for this term in (P1'). Therefore, maximizing the principal's objective function $U(\mathbf{x}, \mathbf{t})$ in (P1) is equivalent to maximizing a new objective function

$$
\int_{\Theta}\{u(\mathbf{x}(\theta), \theta)+\Phi(\mathbf{x}(\theta), \theta)-r(\underline{\theta})\} \mathrm{d} F(\theta)
$$

with the monotonicity condition as the only constraint.

Proof of Proposition 3 Clearly, by condition (1), $X$ is compact and $g$ is upper-semicontinuous in $x$ for each $\theta$. Then $\arg \max _{x \in X} g(x, \theta)$ always exists.

Then, $g$ is supermodular in $x$ and satisfies the single crossing property in $(x, \theta)$. Theorem 2.8.1 in Topkis (1998) implies that any mechanism $\mathbf{x}$ satisfying $\mathbf{x}(\theta) \in \arg \max _{x \in X} g(x, \theta)$ must be increasing.

Proof of Theorem 1 Clearly, $g$ is upper semicontinuous in $x$ for each $\theta$, and therefore Borel-measurable. Thus, $g$ and $G$ will both be real-valued.

Claim 1: $S:=\{x \mid x$ is increasing $\}$ is sequentially compact with the topology of pointwise convergence.

First, for any $\theta_{1}<\theta_{2}$ in $\Theta$, consider any sequence $\left\{\mathbf{x}_{n}\right\}$ in $S$ converging to some $\mathbf{x}$ pointwise. Thus,

$$
\begin{aligned}
\mathbf{x}\left(\theta_{1}\right)-\mathbf{x}\left(\theta_{2}\right) & =\left[\mathbf{x}\left(\theta_{1}\right)-\mathbf{x}_{n}\left(\theta_{1}\right)\right]+\left[\mathbf{x}_{n}\left(\theta_{1}\right)-\mathbf{x}_{n}\left(\theta_{2}\right)\right]+\left[\mathbf{x}_{n}\left(\theta_{2}\right)-\mathbf{x}\left(\theta_{2}\right)\right] \\
& \leq\left\|\mathbf{x}\left(\theta_{1}\right)-\mathbf{x}_{n}\left(\theta_{1}\right)\right\| I+\left\|\mathbf{x}_{n}\left(\theta_{1}\right)-\mathbf{x}_{n}\left(\theta_{2}\right)\right\| I+\left\|\mathbf{x}_{n}\left(\theta_{2}\right)-\mathbf{x}\left(\theta_{2}\right)\right\| I,
\end{aligned}
$$

where $I$ denotes the unit vector $(1, \ldots, 1)$ in the vector space of $X$. Now, letting $n \rightarrow \infty$, we can see that $\mathbf{x}\left(\theta_{1}\right)-\mathbf{x}\left(\theta_{2}\right) \leq 0$. Therefore, $S$ is sequentially closed with the topology of pointwise convergence.

Second, by Theorem 1 in Chistyakov (2005), since Assumption 10 implies that any increasing $\mathbf{x}$ in $S$ must have a bounded variation, any consequence in $S$ must contain a 
convergent subsequence. This subsequence must converge in $S$, since $S$ is sequentially closed. This completes the proof of Claim 1.

Claim 2: By the Lebesgue Dominated Convergence theorem, $G(x)$ is sequentially upper semicontinuous; that is, for any sequence $x_{n}$ convergent to $x, \lim \sup _{n} G\left(x_{n}\right)$ $\leq G(x)$.

Under Assumption 3, Fatou's Lemma implies that

$$
\lim \sup _{x} G\left(\mathbf{x}_{n}\right) \leq \int_{\Theta} \lim \sup _{n} g\left(\mathbf{x}_{n}(\theta), \theta\right) \mathrm{d} F(\theta),
$$

for any sequence $\mathbf{x}_{n}$ convergent to $\mathbf{x} .{ }^{15}$ Since $g$ is upper semicontinuous in $x$ for each $\theta$,

$$
\lim \sup _{n} g\left(\mathbf{x}_{n}(\theta), \theta\right) \leq g(\mathbf{x}(\theta), \theta)
$$

for each $\theta$. Then, Claim 2 is an immediate consequence.

Next, given a sequence $\mathbf{x}_{n}$ converging to $\mathbf{x}$ in $S$, which is clearly nonempty, define $s:=\sup _{\mathbf{x} \in S} G(\mathbf{x})$.

Claim 3: $s$ is finite.

We need to show there exists a real number $a$ such that $G(\mathbf{x}) \leq a$ for all $\mathbf{x}$ in $S$. Suppose not. There thus exists some sequence $\left\{\mathbf{x}_{n}\right\}$ in $S$ such that $G\left(\mathbf{x}_{n}\right)>n$ for all $n \in \mathbb{N}$. By sequential compactness of $S$, there must exist some subsequence $\left\{\mathbf{x}_{n_{m}}\right\}$ of $\left\{\mathbf{x}_{n}\right\}$ in $X$ such that $\mathbf{x}_{n_{m}}$ pointwise converges to some $\mathbf{x}$ in $S$ and $G\left(\mathbf{x}_{n_{m}}\right)>n_{m}$ for all $m \in \mathbb{N}$. Hence, $\lim \sup _{n_{m}} G\left(\mathbf{x}_{n_{m}}\right)$ must be infinity. Sequential upper semi-continuity of $G$ implies that $G(\mathbf{x})=\infty$. This contradicts the fact that $G$ is real-valued, so Claim 3 holds.

Finally, since $s$ is the supremum, there must exist a sequence $\left\{\mathbf{x}_{n}\right\}$ in $S$ such that $G\left(\mathbf{x}_{n}\right) \rightarrow s$, as $n \rightarrow \infty$. By sequential compactness of $S$, there must exist a subsequence $\left\{\mathbf{x}_{n_{m}}\right\}$ of $\left\{\mathbf{x}_{n}\right\}$ in $S$ such that $\mathbf{x}_{n_{m}}$ pointwise converges to some $\mathbf{x}^{*}$ in $S$ and $G\left(\mathbf{x}_{n_{m}}\right) \rightarrow s$, as $n \rightarrow \infty$. Finally, sequential upper semi-continuity of $G$ implies that $\lim \sup _{n_{m}} G\left(\mathbf{x}_{n_{m}}\right) \leq G\left(\mathbf{x}^{*}\right)$. Note that $\lim \sup _{n_{m}} G\left(\mathbf{x}_{n_{m}}\right)=s$, since $G\left(\mathbf{x}_{n_{m}}\right) \rightarrow s$, as $n \rightarrow \infty$. Therefore, $G\left(\mathbf{x}^{*}\right)=s$.

\section{References}

Aliprantis, C.D., Border, K.C.: Infinite Dimensional Analysis: A Hitchhiker's Guide. Springer, Berlin (2006) Apostol, T.M.: Mathematical Analysis, vol. 2, 2nd edn. Addison-Wesley, Reading (1974)

Bolton, P., Dewatripont, M.: Contract Theory. MIT Press, Cambridge (2005)

Carlier, G.: A general existence result for the principal-agent problem with adverse selection. J. Math. Econ. 35(1), 129-150 (2001)

Chistyakov, V.V.: The optimal form of selection principles for functions of a real variable. J. Math. Anal. Appl. 310(2), 609-625 (2005)

Dequiedt, V., Martimort, D.: Vertical contracting with informational opportunism. Am. Econ. Rev. 105(7), 2141-2182 (2015)

Guesnerie, R., Laffont, J.J.: A complete solution to a class of principal-agent problems with an application to the control of a self-managed firm. J. Public Econ. 25(3), 329-369 (1984)

$\overline{15}$ This holds if we replace Assumption 3 with Assumptions 6 and 7. 
Hart, O.D.: Optimal labour contracts under asymmetric information: an introduction. Rev. Econ. Stud. 50(1), 3-35 (1983)

Kadan, O., Reny, P.J., Swinkels, J.M.: Existence of optimal mechanisms in principal-agent problems. Econometrica 85(3), 769-823 (2017)

Koliha, J.J.: A fundamental theorem of calculus for Lebesgue integration. Am. Math. Mon. 113(6), 551-555 (2006)

Laffont, J.J., Martimort, D.: The Theory of Incentives: The Principal-Agent Model. Princeton University Press, Princeton (2009)

Maskin, E., Riley, J.: Monopoly with incomplete information. RAND J. Econ. 15(2), 171-196 (1984)

Milgrom, P., Segal, I.: Envelope theorems for arbitrary choice sets. Econometrica 70(2), 583-601 (2002)

Page, F.H.: Optimal contract mechanisms for principal-agent problems with moral hazard and adverse selection. Econ. Theory 1(4), 323-338 (1991)

Page, F.H.: Mechanism design for general screening problems with moral hazard. Econ. Theory 2(2), 265-281 (1992)

Stole, L.: Lectures on the theory of contracts and organizations. Unpublished Monograph (2001)

Tadelis, S., Segal, I.: Lectures in Contract Theory, Lecture Notes for UC Berkeley and Stanford University (2005)

Topkis, D.M.: Supermodularity and Complementarity. Princeton University Press, Princeton (1998)

Ülkü, L.: Optimal combinatorial mechanism design. Econ. Theory 53(2), 473-498 (2013)

Publisher's Note Springer Nature remains neutral with regard to jurisdictional claims in published maps and institutional affiliations. 